\title{
Impacto diferencial de la religión en el prejuicio entre muestras cristianas y musulmanas
}

\section{Differential impact of religion on prejudice in Muslim and Christian samples}

\author{
Máximo Núñez Alarcón, Félix Moral Toranzo y Mª del Pilar Moreno Jiménez
}

Universidad de Málaga, España

Disponible online 30 de diciembre de 2010

\begin{abstract}
El objetivo de este trabajo es avanzar en la investigación que analiza la asociación entre religión y prejuicio en cristianos y musulmanes, dentro y fuera del contexto universitario. Este estudio se ha realizado con 211 cristianos y 121 musulmanes universitarios y 155 cristianos y 220 musulmanes no universitarios. Se ha utilizado un instrumento de orientación religiosa así como diferentes medidas de religión subjetiva, prejuicio religioso, autoritarismo de derechas, fundamentalismo, frecuencia de oración, dogmatismo, literalismo, creencias, emociones, favorabilidad y racismo moderno. Los resultados obtenidos son los siguientes: 1) En líneas generales, ser musulmán y/o no universitario conlleva ser más religioso, fundamentalista, autoritario e interpretar más literalmente los textos sagrados. 2) Las variables que mejor predicen el prejuicio en la muestra cristiana son el autoritarismo de derechas y el fundamentalismo. En la muestra musulmana emergen como predictores el dogmatismo y las orientaciones religiosas extrínseca e intrínseca. 3) Los cristianos puntúan más alto en la orientación de búsqueda, que se manifiesta como una dimensión distinta al resto de las orientaciones y variables religiosas.
\end{abstract}

Palabras clave: Prejuicio; Religión; Islam; Cristianismo.

The aim of the present paper was to build on previous research that examined the association between religion and prejudice in Christians and Muslims from inside and outside the university context. The study included 211 Christian and 121 Muslim university students and 155 Christian and 220 Muslim non-students. An instrument for evaluating religious orientation was used as well as various measures of subjective religion, religious prejudice, right-wing authoritarianism, fundamentalism, frequency of prayer, dogmatism, literalism, beliefs, emotions, favourability and modern racism. The results were as follows: 1) in general, Muslims and/or non-university students were more likely to be more religious, fundamentalist, and authoritarian and to interpret religious texts more literally; 2) the variables that best predicted prejudice in the Christian sample were right-wing authoritarianism and fundamentalism. The predictors of prejudice in the Muslim sample were dogmatism and extrinsic and intrinsic religious orientations; 3) the Christian sample scored higher in the quest dimension which manifested as a dimension different from other religious orientations and variables.

Keywords: Prejudice; Religion; Islam; Christianity.

Correspondencia: Máximo Núñez Alarcón. c/ Encarnación Fontiveros, 5, 10E, 29010-Málaga, España. Fax: (+34) 9521311 00. E-mail: nmaximo7@gmail.com. E-Mail de los otros autores: Félix Moral Toranzo: fmoral@uma.es, Ma del Pilar: Moreno: Jiménez mpilar@uma.es. 
La relación de la religión con el prejuicio es compleja. A pesar de que las enseñanzas de la mayoría de las confesiones religiosas promueven el respeto y la tolerancia, Batson, Schoenrade y Ventis (1993) ponen de manifiesto que, entre 1940 y 1990, de 47 estudios, 37 presentan una relación positiva entre religiosidad y prejuicio y sólo dos, una relación negativa. En este sentido, Wulff (1997) muestra que medidas de religiosidad como la afiliación religiosa, la asistencia a la iglesia, la ortodoxia doctrinal, la importancia de la religión, etc., correlacionan positivamente con etnocentrismo, autoritarismo, dogmatismo, distancia social y prejuicio hacia negros y judíos. Además, numerosos estudios confirman la hipótesis de que cuanto más religiosa es una persona, mayor probabilidad tiene de ser prejuiciosa (Meadow y Kahoe, 1985).

Tradicionalmente, se ha considerado que existen dos dimensiones religiosas (Allport y Ross, 1967). Una extrínseca caracterizada por el hecho de encontrar la religión útil por ofrecer a los creyentes seguridad, consuelo, distracción, estatus y auto justificación; características que provienen de una fe inmadura y una orientación más externa y utilitaria de la religión. En el otro extremo, la dimensión intrínseca es propia de personas con una fe sincera, interiorizada y madura. Estos autores concluyen: 1) en promedio, las personas que asisten regularmente a la Eucaristía son más prejuiciosas que las que no asisten; 2) una minoría significativa, los intrínsecos, es menos prejuiciosa; y 3) son los miembros ocasionales e irregulares, los extrínsecos, los que marcan alto en prejuicio. Concluyen que la orientación intrínseca correlaciona negativamente con el prejuicio étnico, y la orientación extrínseca, positivamente. Sin embargo, tanto los instrumentos que utilizaron como el enlace entre dichas orientaciones y el prejuicio han sido criticados y revisados (Altemeyer, 1996). Batson et al. (1993) afirman que las dimensiones son independientes, no están relacionadas y no son intercambiables.

Otra dimensión religiosa es la de búsqueda, definida como el grado en que la religión individual implica un diálogo abierto y dinámico con las grandes preguntas existenciales que surgen en las contradicciones y tragedias de la vida (Batson y Schoenrade, 1991). Se ha asociado con el concepto de compasión universal y tolerancia, muestra ser sensible a formas progresistas de religiosidad e incluso puede reflejar cierto desinterés y/o rechazo hacia la religión (Watson, Morris, Hood, Miller y Waddell, 1999). Por otra parte, la orientación de búsqueda correlaciona negativamente con el prejuicio (Batson et al., 1993).

Diversos estudios han vinculado ciertas formas de religiosidad y el fundamentalismo religioso tanto con el prejuicio como con el autoritarismo de derechas (Laythe, Finkel, Bringle y Kirkpatrick, 2002). En países musulmanes se ha estudiado la relación entre religiosidad y ciertos comportamientos y actitudes políticas. Encuestas en Egipto, Kuwait y Palestina demuestran que ni la piedad islámica ni el soporte al Islam político tienen un impacto en las actitudes hacia la democracia. La frecuencia en la asistencia a la mezquita y el apoyo al Islam político tampoco son significativos para explicar las actitudes hacia la democracia en Argelia, Egipto, Jordania y Marruecos (Tessler, 2003). Rizzo, Abdel-Latif y Mejer (2007) encuentran que, en sociedades musulmanas no árabes, las actitudes hacia la igualdad de género y el divorcio predicen el apoyo a la democracia, pero no el hecho de ser religioso.

Por otra parte, Hunsberger y Jackson (2005) realizan una recopilación de estudios desde 1990 y muestran que la orientación intrínseca, extrínseca y especialmente el fundamentalismo religioso, están asociados con la intolerancia, mientras que la orientación de búsqueda aparece relacionada con la tolerancia. Según los autores, la tesis original de que la orientación intrínseca implica actitudes menos prejuiciosas respecto a la extrínseca puede deberse a un sesgo en las investigaciones, que sólo consideran el prejuicio étnico; así, la escala intrínseca fue relacionada negativamente con la intolerancia racial, pero positivamente con la intolerancia hacia gays y lesbianas. La orientación extrínseca fue positivamente asociada con la intolerancia étnica y el prejuicio hacia gays y lesbianas. La orientación de búsqueda mostró una asociación débil con la tolerancia hacia grupos raciales, apareciendo un efecto positivo mayor cuando el objeto de prejuicio eran gays y lesbianas. Finalmente, el fundamentalismo mostró consistentemente una relación con un incremento en el prejuicio hacia gays y lesbianas, mujeres, comunistas y exogrupos religiosos, así como con el autoritarismo.

¿Cuál puede ser la razón de tal disparidad según el objeto de prejuicio? Muchos tipos de prejuicios están proscritos por muchas religiones, pero otros están tolerados, justificados e incluso potenciados; por ejemplo, el prejuicio hacia gays y lesbianas (Hood, Spilka, Hunsberger y Gorsuch, 2009). Además, en el meta-análisis de Whitley (2009) se afirma que las variables de religiosidad: fundamentalismo, frecuencia de asistencia a los servicios religiosos, ortodoxia, religiosidad subjetiva y orientación intrínseca presentan, al menos, una relación negativa débil con las actitudes hacia gays y lesbianas. La orientación de búsqueda se asocia con actitudes positivas hacia gays y lesbianas y la orientación extrínseca no presenta relación alguna con dichas actitudes. Con una muestra de 1500 americanos adultos, Rowatt, LaBouff, Johnson, Froese y Tsang (2009) apoyan la hipótesis de intolerancia selectiva: la religiosidad se asocia con actitudes más negativas hacia los homosexuales, siendo despreciable su relación con el prejuicio racial. Se ha argumentado que la dimensión intrínseca está relacionada con la antipatía hacia los que rompen los valores, es decir, los gays o los heterosexuales promiscuos, pero no hacia los gays como individuos (Tsang y Mak, 2008). Sin embargo, otros estudios encuentran una relación clara entre la dimensión intrínseca y el prejuicio hacia los homosexuales, incluso controlando estadísticamente el autoritarismo de derechas (Tsang y Rowatt, 2007).

En sus conclusiones, Hunsberger y Jackson (2005) lamentan que existen pocos estudios entre las distintas orientaciones religiosas y el prejuicio cuando los objetos de éste son las mujeres, los comunistas y los exogrupos religiosos. Además, son escasos los estudios transculturales que estudien diversas 
religiones o en los que se realicen comparaciones transculturales. Estos argumentos sugieren avanzar en la investigación sobre el binomio religión-prejuicio comparando muestras cristianas y musulmanas en un marco intercultural, así como crear un instrumento, Escala del Prejuicio religioso, que integre los diferentes grupos hacia los que parece que se dirige el prejuicio de los religiosos. Se llevan a cabo sendos estudios, uno con universitarios y otro realizado en centros de culto, para conocer las relaciones entre las distintas orientaciones y variables religiosas con el prejuicio, así como, determinar si existen diferencias en función del credo (cristianos versus musulmanes) y del grupo (universitario versus centros de culto). Nuestra hipótesis es que las distintas orientaciones y variables religiosas van a correlacionar entre sí y con el prejuicio religioso. Además, tanto musulmanes como no universitarios serán más religiosos, fundamentalistas y autoritarios de derechas e interpretarán más literalmente los textos sagrados.

\section{Método}

\section{Participantes}

Se han llevado a cabo dos estudios y en ambos se han formado dos grupos. En el primero, la muestra cristiana está constituida por 211 estudiantes: $28.40 \%$ hombres $\left(M_{\text {edad }}=21.59\right.$, $D T=3.24)$ y $71.26 \%$ mujeres ( $M_{\text {edad }}=20.67, D T=3.27$ ) y la muestra musulmana está formada por 121 estudiantes: $60 \%$ hombres $\left(M_{\text {edad }}=25.58, D T=15.58\right)$ y $36.80 \%$ mujeres $\left(M_{\text {edad }}=24.80\right.$, $D T=5.1)$. El segundo estudio se ha realizado con sujetos procedentes de grupos religiosos, parroquias y mezquitas. La muestra cristiana consta de 155 participantes: $40.60 \%$ hombres $\left(M_{\text {edad }}=42.73, D T=21.12\right)$ y $58.70 \%$ mujeres $\left(M_{\text {edad }}=42.97\right.$, $D T=20.44)$ y la muestra musulmana de un total de 220 participantes de los cuales, $51.60 \%$ eran hombres $\left(M_{\text {edad }}=35.19\right.$, $D T=12.71)$ y $45.80 \%$ mujeres $\left(M_{\text {edad }}=36.03, D T=12.48\right)$.

\section{Procedimiento}

El cuestionario fue administrado de forma personal en muestras de conveniencia obtenidas en los campus universitarios de Málaga, Melilla y Granada identificadas fuera de las aulas y en los centros de culto, parroquias y mezquitas de Málaga. Paralelamente, se ha utilizado la técnica bola de nieve (Taylor y Bodgan, 1986) para los cuatro subgrupos realizados.

\section{Instrumentos}

Se ha diseñado un cuestionario en el que se han traducido los instrumentos de los que no había disponible ninguna adaptación al castellano. Está compuesto de las siguientes escalas:

a) Racismo moderno. Se utiliza la versión de la Escala de Racismo Moderno traducida y adaptada al español por García, Navas, Cuadrado y Molero (2003). Es una escala compuesta por 10 ítems, las puntuaciones oscilan entre 1 y 7, una mayor puntuación implica un grado mayor de racismo moderno. La fiabilidad de las diferentes escalas se presenta en la Tabla 1.

b) Dimensiones afectivas y cognitivas del prejuicio. Se incluyen diferentes medidas de las principales categorías del prejuicio (Tropp y Pettigrew, 2005): dimensiones afectivas (emoción y favorabilidad) y cognitivas (creencias). La emoción se mide con Emotions Scale de Stephan y Stephan (1985). Siguiendo las directrices de Tropp y Pettigrew (2005) se utilizan dos variables: emociones positivas y negativas. Con otros dos ítems se mide la favorabilidad hacia el exogrupo, Favorability Scale (Tropp y Pettigrew, 2005). Las escalas Whites'Attitudes Toward Black y Blacks'Attitudes Toward White de Brigham (1993) se utilizaron para medir las creencias y evaluar no sólo las actitudes de los blancos hacia los negros sino también las de los negros hacia los blancos. La adaptación realizada consiste en la utilización de la religión como forma de categorización en vez del color de la piel: la categoría "negro" se ha sustituido por "musulmán” y "blanco" por "cristiano".

c) Autoritarismo de derechas. Se utiliza la versión de Manganelli Rattazzi, Bobbio y Canova (2007). Esta escala consta de 14 ítems y dos factores: agresión autoritaria y sumisión y conservadurismo, éste último está medido en sentido inverso.

d) Orientación religiosa intrínseca, extrínseca y de búsqueda. Se ha utilizado una adaptación española de Ramírez de la Fe (2006) de 31 ítems de la escala de orientación religiosa de Batson y Ventis (1982).

e) Oración personal. Se ha incluido un ítem relativo a la frecuencia de la oración personal para realizar una distinción más clara entre la expresión personal y pública de la religión (Maltby, Lewis y Day, 1999).

f) Dogmatismo. Se utiliza un ítem empleado en varias encuestas del Centro de Investigaciones sobre la Realidad Social CIRES (1993).

g) Fundamentalismo religioso. Se recurre a la escala de Altemeyer. y Hunsberger (2004) de 12 ítems.

h) Visión conservadora teológica o literalismo de Gay y Lynxwiler (1999). Se trata de un único ítem medido en sentido inverso.

i) Religión subjetiva. Seguimos las indicaciones de Mattis, Fontenot y Hatcher-Kay (2003) que toman como base los instrumentos de Levin, Taylor y Chatters (1995) y Taylor, Mattis y Chatters (1999) para medir la religión subjetiva manifestada por los propios sujetos a través de tres ítems.

j) Prejuicio religioso. Medido con una escala tipo Likert que oscila entre 1 y 7. Puntuaciones más altas indican un mayor grado de prejuicio religioso. Contiene 14 ítems distribuidos en cuatro factores: homofobia (Ellis, Kitzinger y Wilkinson, 2002; Herek, 1998), intolerancia religiosa (Aosved y Long, 2006), sexismo (Glick y Fiske, 1996) y distancia social (Brighman, 1993). El instrumento ha sido validado tanto para una muestra cristiana universitaria $\left(\alpha_{\text {Cristiana }}=.83\right)$ como no universitaria $\left(\alpha_{\mathrm{No}-}\right.$ cristiana $=.75$ ). 
Tabla 1. Coeficiente Alfa de Cronbach de las distintas escalas.

\begin{tabular}{|c|c|c|c|c|c|}
\hline \multirow{2}{*}{ Escala } & \multirow{2}{*}{ Original } & \multicolumn{2}{|c|}{ Musulmanes } & \multicolumn{2}{|c|}{ Cristianos } \\
\hline & & Universitarios & No Universitario & Universitarios & No Universitario \\
\hline Racismo Moderno & .72 & .75 & .70 & .84 & .84 \\
\hline Emociones Positivas & .91 & .86 & .80 & .90 & .96 \\
\hline Emociones Negativas & .84 & .88 & .94 & .86 & .96 \\
\hline Favorabilidad & .84 & .77 & .89 & .82 & .89 \\
\hline Creencias & $.89 / 75^{1}$ & .85 & .84 & .89 & .84 \\
\hline Agresión autoritaria & .72 & .72 & .74 & .84 & .90 \\
\hline Conservadurismo & .75 & .79 & .74 & .76 & .76 \\
\hline Intrínseca & .89 & .81 & .84 & .85 & .92 \\
\hline Extrínseca & .72 & .74 & .65 & .73 & .71 \\
\hline Búsqueda & .73 & .81 & .80 & .75 & .77 \\
\hline Fundamentalismo & .91 & .80 & .80 & .86 & .84 \\
\hline Religión subjetiva & .88 & .75 & .58 & .75 & .91 \\
\hline
\end{tabular}

${ }^{1}$ En Creencias se considera la $\alpha$ de la escala de actitudes de los blancos hacia los negros y el de los negros respecto a los blancos respectivamente.

Resultados

En ambos estudios no se encuentran diferencias significativas por género y, por tanto, se omite esta variable del artículo.

Estudio $1^{\mathrm{o}}$ : Muestra universitaria

\section{Muestra universitaria cristiana}

Se realiza un estudio correlacional (Tabla 2) y se encuentra que las siguientes variables correlacionan positivamente: religión subjetiva, orientación intrínseca, frecuencia de oración, fundamentalismo, autoritarismo, literalismo y conservadurismo. La orientación de búsqueda, si bien correlaciona con la orientación intrínseca y la extrínseca, no lo hace con la frecuencia de oración ni con la sumisión y la agresión autoritaria, mientras que correlacio na negativamente con el fundamentalismo religioso y con el conservadurismo.
Existe una correlación positiva débil $(\mathrm{p}<.05)$ entre religión subjetiva y prejuicio religioso. Este resultado se replica con la orientación intrínseca. La orientación extrínseca correlaciona positiva y débilmente sólo con la homofobia pero no con ningún otro factor ni con el prejuicio religioso. Una correlación más fuerte se encuentra entre el prejuicio religioso y los factores que lo componen con el fundamentalismo, el autoritarismo y el conservadurismo.

Las orientaciones extrínseca e intrínseca no correlacionan con ninguna otra medida del prejuicio. Sin embargo, la orientación de búsqueda correlaciona positivamente con creencias y negativamente con racismo moderno, intolerancia religiosa y prejuicio religioso, mostrando que las personas con orientación de búsqueda son menos prejuiciosas. La sumisión y agresión autoritaria correlaciona negativamente con creencias, emociones positivas y favorabilidad; y positivamente con conservadurismo, racismo moderno, emociones negativas, homofobia, sexismo, intolerancia religiosa, distancia social y prejuicio religioso. El conservadurismo mantiene, por lo general, estas correlaciones.

Tabla 2. Correlaciones de la muestra cristiana universitaria.

\begin{tabular}{|c|c|c|c|c|c|c|c|c|c|c|c|c|c|c|c|c|}
\hline & 2 & 3 & 4 & 5 & 6 & 7 & 8 & 9 & 10 & 11 & 12 & 13 & 14 & 15 & 16 & Extrínseca \\
\hline 1 Intrínseca & $.22 * *$ & $.68 * * *$ & $.18^{* *}$ & $.31 * *$ & $.61 * * *$ & $.62 * * *$ & .12 & $.31 * * *$ & .04 & .13 & $.16^{*}$ & $-.42 * * *$ & $.15^{*}$ & -.05 & .03 & $.41 * * *$ \\
\hline 2 Búsqueda & & .00 & -.12 & $.24 * *$ & $.18^{*}$ & .04 & $-.16^{*}$ & -.06 & -.04 & -.12 & .06 & -.03 & $-.16^{*}$ & $.17^{*}$ & .46 & $.33 * * *$ \\
\hline 3 Religión subjetiva & & & $.14 *$ & $-.34 * * *$ & $.59 * * *$ & $.57 * * *$ & .03 & $.31 * * *$ & .08 & .08 & .11 & $-.41 * * *$ & $.14 *$ & .01 & .06 & $.39 * * *$ \\
\hline 4 Autoritarismo & & & & $-.29 * * *$ & $.38^{* * *}$ & .06 & $.45^{* * *}$ & $.31 * * *$ & $.47 * * *$ & $.27 * * *$ & $.34 * * *$ & $-.22 * *$ & $.50 * * *$ & $-.36 * * *$ & $-.22 * *$ & $.15^{*}$ \\
\hline 5 Conservadurismo & & & & & $-.55^{* * *}$ & -.10 & $-.44 * * *$ & $-.60 * * *$ & -.12 & $-.49 * *$ & $-.24 * * *$ & $.31 * * *$ & $-.50 * * *$ & $.44 * * *$ & $.21 * *$ & -.12 \\
\hline 6 Fundamentalismo & & & & & & $.37 * * *$ & $.26 * * *$ & $.46 * * *$ & $.17 *$ & $.25 * * *$ & $.26 * * *$ & $-.52 * * *$ & $.35 * * *$ & $-.21 * *$ & -.10 & $.26 * * *$ \\
\hline 7 Oración & & & & & & & .11 & .07 & -.03 & -.00 & .10 & $-.35 * *$ & .04 & -.01 & .05 & $.32 * * *$ \\
\hline 8 Racismo Moderno & & & & & & & & $.37 * *$ & $.26 * *$ & $.58 * *$ & $.25 * *$ & $-.10 *$ & $.60 * *$ & $-.74 * * *$ & $-.39 * * *$ & .08 \\
\hline 9 Homofobia & & & & & & & & & $.20 * *$ & $.43 * *$ & $.21 * *$ & $-.29 * *$ & $.65^{* *}$ & $-.41 * * *$ & $-.14^{*}$ & $.14^{*}$ \\
\hline 10 Sexismo & & & & & & & & & & $.24 * *$ & $.33 * *$ & -.11 & $.67 * *$ & $-.27 * * *$ & $-.16^{*}$ & .04 \\
\hline 11 Distancia & & & & & & & & & & & $.20^{* *}$ & -.13 & $.79 * *$ & $-.88 * * *$ & $-.58 * * *$ & .07 \\
\hline 12 Dogmatismo & & & & & & & & & & & & $-.27 * *$ & $.32 * *$ & $-.16^{*}$ & -.09 & .12 \\
\hline 13 Literalismo & & & & & & & & & & & & & $-.19 * *$ & .09 & .07 & $-.36^{* * *}$ \\
\hline 14 Prejuicio Religioso & & & & & & & & & & & & & & $-.79 * *$ & $-.48^{* * *}$ & .06 \\
\hline 15 Creencias & & & & & & & & & & & & & & & $.60^{* *}$ & -.06 \\
\hline 16 Favorabilidad & & & & & & & & & & & & & & & & -.08 \\
\hline
\end{tabular}

${ }^{*} p<.05 ; * * p<.01 ;{ }^{* * *} p<.001$. 
Al realizar un análisis de regresión lineal, se observa que las variables religiosas (intrínseca, extrínseca, búsqueda, religión subjetiva, frecuencia de oración y literalismo) tienen poco o ningún valor predictivo. Sólo el fundamentalismo y el conservadurismo predicen la homofobia con una bondad del ajuste del modelo superior al 0.20. La variable que mejor predice el prejuicio medido con la variable de creencias es el conservadurismo $\left(R^{2}=.19\right)$. Este resultado se repite en racismo moderno, sólo que se agrega como predictor la sumisión y agresión autoritaria.

\section{Muestra universitaria musulmana}

En esta muestra se encuentra que las orientaciones extrínseca, intrínseca y la religión subjetiva correlacionan tanto entre sí como con fundamentalismo, autoritarismo, conservadurismo, frecuencia de oración y literalismo (Tabla 3). Las orientaciones extrínseca, intrínseca y de búsqueda correlacionan positivamente con creencias y favorabilidad y negativamente con el racismo moderno, es decir, las personas que se manifiestan más religiosas en estas dimensiones son menos prejuiciosas hacia los cristianos. Las distintas orientaciones y variables (intrínseca, extrínseca, religión subjetiva, fundamentalismo, autoritarismo, conservadurismo y literalismo) correlacionan positivamente con la homofobia y el sexismo. En cambio, la orientación de búsqueda no correlaciona con la homofobia y lo hace, pero sólo débilmente, con el sexismo. De forma opuesta a sus pares cristianos, la sumisión autoritaria y el conservadurismo no correlacionan con creencias, emociones, favorabilidad ni con racismo moderno.

Tabla 3. Correlaciones de la muestra musulmana universitaria.

\begin{tabular}{|c|c|c|c|c|c|c|c|c|c|c|c|c|c|c|c|}
\hline & 2 & 3 & 4 & 5 & 6 & 7 & 8 & 9 & 10 & 11 & 12 & 13 & 14 & 15 & Extrínseca \\
\hline 1 Intrínseca & .08 & $.46^{* * *}$ & $.46^{* * *}$ & $.56 * * *$ & $.66^{* * *}$ & $.38 * * *$ & $-.20 *$ & $.52 * * *$ & $.40 * * *$ & $-.25 * *$ & -.14 & $-.51 * * *$ & $.29 * *$ & $.18^{*}$ & $.46^{* * *}$ \\
\hline 2 Búsqueda & & -.16 & -.11 & .06 & -.02 & .01 & $-.28 * *$ & .06 & $.20 *$ & -.08 & -.14 & -.05 & $.24 * *$ & $.19 *$ & $.35 * * *$ \\
\hline 3 Religión subjetiva & & & $.23 *$ & $-.61 * * *$ & $.54 * * *$ & $.67^{* * *}$ & .00 & $.40 * * *$ & $.30 * *$ & .10 & .10 & $-.34 * * *$ & .06 & .00 & $.39 * * *$ \\
\hline 4 Autoritarismo & & & & $-.43 * * *$ & $.57 * * *$ & .09 & .07 & $.34 * * *$ & $.29 * *$ & .05 & $.29 * *$ & $.34 * * *$ & .14 & -.08 & $.29 * *$ \\
\hline 5 Conservadurismo & & & & & $-.65^{* * *}$ & $-.59^{* * *}$ & -.01 & $-.61^{* * *}$ & $-.24 * *$ & -.11 & -.08 & $.41 * * *$ & -.07 & .00 & $-.46^{* * *}$ \\
\hline 6 Fundamentalismo & & & & & & $.47 * * *$ & -.12 & $.66^{* * *}$ & $.55^{* * *}$ & -.05 & .00 & $-.60 * * *$ & .18 & .10 & $.51 * * *$ \\
\hline 7 Oración & & & & & & & -.10 & $.39 * *$ & $.35^{* *}$ & .10 & -.10 & $-.25 *$ & .11 & .07 & $.37 * * *$ \\
\hline 8 Racismo Moderno & & & & & & & & $-.31 * *$ & $-.34 * *$ & $.51 * *$ & $.45^{* *}$ & .11 & $-.71 * * *$ & $-.66 * * *$ & $-.37 * * *$ \\
\hline 9 Homofobia & & & & & & & & & $.50 * *$ & -.08 & -.13 & $-.50 * *$ & .17 & .02 & $.44 * * *$ \\
\hline 10 Sexismo & & & & & & & & & & $-.26^{*}$ & -.06 & $-.42 * *$ & $.31 * * *$ & $.26^{* *}$ & $.42 * * *$ \\
\hline 11 Distancia & & & & & & & & & & & $.34 * *$ & .05 & $-.78 * * *$ & $-.51 * * *$ & -.09 \\
\hline 12 Dogmatismo & & & & & & & & & & & & .04 & $-.51 * * *$ & $-.49 * * *$ & -.08 \\
\hline 13 Literalismo & & & & & & & & & & & & & -.13 & .01 & $-.33 * *$ \\
\hline 14 Creencias & & & & & & & & & & & & & & $.68 * *$ & $.31 * *$ \\
\hline 15 Favorabilidad & & & & & & & & & & & & & & & $.28^{* *}$ \\
\hline
\end{tabular}

$* p<.05 ; * * p<.01 ; * * * p<.001$.

En el análisis de regresión lineal sólo el dogmatismo y la orientación extrínseca demuestran cierta capacidad predictiva relativa al prejuicio tanto medido con la variable de racismo moderno $\left(R^{2}=.27\right.$ y $\left.R^{2}=.13\right)$ como con creencias $\left(R^{2}=.14\right.$ y $\left.R^{2}=.10\right)$.

\section{Diferencias entre cristianos y musulmanes universitarios}

Comparando ambas muestras, los musulmanes son significativamente menos prejuiciosos en varias medidas (emociones positivas, negativas y favorabilidad) pero más homófobos y sexistas (Tabla 4).

Tabla 4. Diferencias de medias por religión y grupo.

\begin{tabular}{|c|c|c|c|c|c|c|c|c|}
\hline & \multicolumn{4}{|c|}{ Universitario } & \multicolumn{4}{|c|}{ No Universitario } \\
\hline & \multicolumn{2}{|c|}{ Cristiano } & \multicolumn{2}{|c|}{ Musulmán $^{1}$} & \multicolumn{2}{|c|}{ Cristiano } & \multicolumn{2}{|c|}{ Musulmán ${ }^{2}$} \\
\hline & $M$ & $S D$ & $M$ & $S D$ & $M$ & $S D$ & $M$ & $S D$ \\
\hline Racismo Moderno & 3.05 & .95 & $3.51(* * *)$ & .87 & 2.63 & 1.03 & $3.30(*)$ & .85 \\
\hline Emociones Negativas & 2.71 & 1.13 & $1.85(* * *)$ & 1.07 & 2.47 & 1.48 & 2.05 & 1.03 \\
\hline Creencias & 3.52 & .59 & 3.64 & .56 & 2.89 & .58 & $3.46(* *)$ & .56 \\
\hline Oración & 2.72 & 1.18 & $4.08(* * *)$ & 1.25 & 4.05 & 1.24 & $4.53(* * *)$ & .80 \\
\hline Intrínseca & 4.30 & 1.59 & $6.99(* * *)$ & 1.51 & 6.63 & 1.99 & 7.31 & 1.08 \\
\hline Religión Subjetiva & 2.22 & .65 & $3.58(* * *)$ & .90 & 3.32 & 1.10 & 3.67 & .79 \\
\hline Literalismo & 2.22 & .47 & $1.27(* * *)$ & .47 & 2.03 & .44 & $1.22(* * *)$ & .43 \\
\hline Fundamentalismo & 3.22 & 1.08 & $5.72(* * *)$ & 1.30 & 4.86 & 1.46 & $6.64(* * *)$ & 1.01 \\
\hline Sumisión y agresión & 3.79 & 1.23 & $4.41(* * *)$ & 1.02 & 4.64 & 1.63 & $5.17(* * *)$ & .98 \\
\hline Conservadurismo & 5.42 & .87 & $3.80(* * *)$ & 1.06 & 4.50 & 1.19 & $3.23(* * *)$ & 1.07 \\
\hline Homofobia & 2.17 & 1.22 & $4.93(* * *)$ & 1.52 & 1.89 & 2.84 & $4.46(*)$ & 2.01 \\
\hline Sexismo & 4.33 & 1.12 & $4.96(* * *)$ & 1.04 & 4.78 & 1.28 & $4.47(* * *)$ & 1.35 \\
\hline
\end{tabular}

${ }^{1}$ Diferencias de medias entre cristianos y musulmanes universitarios. ${ }^{2}$ Diferencias de medias entre cristianos y musulmanes extraídos de centros de culto. ${ }^{*} p<.05 ; * * p<.01 ; * * * p<.001$. 
Por otra parte, los musulmanes se muestran de forma significativa más religiosos en la dimensión extrínseca, intrínseca, religión subjetiva y frecuencia de oración, interpretan más literalmente las sagradas escrituras y obtienen mayor puntuación en fundamentalismo, autoritarismo y conservadurismo. Los cristianos, en cambio, puntúan más alto en la orientación de búsqueda.

Estudio $2^{\circ}$ : Muestra extraída de centros de culto

\section{Muestra cristiana}

Al realizar un estudio correlacional se comprueba que la orientación intrínseca y las variables siguientes: religión sub- jetiva, fundamentalismo religioso, sumisión y agresión autoritaria, frecuencia de oración, prejuicio religioso, literalismo y conservadurismo, están correlacionadas positivamente considerando que estas dos últimas están medidas en sentido inverso (Tabla 5).

Cabe resaltar que la orientación intrínseca correlaciona positivamente con favorabilidad y negativamente con el racismo moderno. La dimensión extrínseca se comporta de forma distinta al estudio anterior y no correlaciona con la dimensión intrínseca, de búsqueda ni con el prejuicio religioso. La dimensión de búsqueda presenta correlaciones positivas con creencias y favorabilidad y negativa con el racismo moderno, es decir, correlaciona negativamente con el prejuicio.

Tabla 5. Correlaciones de la muestra cristiana extraída de centros de culto.

\begin{tabular}{|c|c|c|c|c|c|c|c|c|c|c|c|c|c|c|c|c|}
\hline & 2 & 3 & 4 & 5 & 6 & 7 & 8 & 9 & 10 & 11 & 12 & 13 & 14 & 15 & 16 & Extrínseca \\
\hline 1 Intrínseca & $.28 * * *$ & $.85 * * *$ & $.47 * * *$ & $.36 * * *$ & $.68 * * *$ & $.85 * * *$ & $-.21 * *$ & $.32 * * *$ & $.35 * * *$ & .13 & $.16^{*}$ & $-.49 * * *$ & $.28 * * *$ & .01 & $.26^{* *}$ & .09 \\
\hline 2 Búsqueda & & .11 & .01 & $.34 * * *$ & -.05 & .12 & $-.19 *$ & -.09 & $.23^{* *}$ & -.10 & .06 & -.01 & -.05 & $.20 *$ & $.20 *$ & .04 \\
\hline 3 Religión subjetiva & & & $.48 * * *$ & $.42 * * *$ & $.66^{* * *}$ & $.81 * * *$ & .12 & $.35 * * *$ & $.34 * * *$ & $.17 *$ & .16 & $-.42 * * *$ & $.34 * * *$ & -.07 & $.17 *$ & -.05 \\
\hline 4 Autoritarismo & & & & $.46 * * *$ & $.72 * * *$ & $.50 * * *$ & .01 & $.38 * * *$ & $.60 * *$ & $.36^{* * *}$ & $.33 * * *$ & $-.25 * *$ & $.61 * * *$ & $-.37 * * *$ & .01 & $.17 *$ \\
\hline 5 Conservadurismo & & & & & $-.66^{* * *}$ & $-.49 * * *$ & -.11 & $-.49 * * *$ & -.12 & $-.34 * * *$ & -.06 & $.40 * * *$ & $-.46^{* * *}$ & $.42 * * *$ & $.16^{*}$ & $-.20 *$ \\
\hline 6 Fundamentalismo & & & & & & $.72 * * *$ & .13 & $.46 * * *$ & $.42 * * *$ & $.31 * * *$ & .13 & $-.48 * * *$ & $.52 * * *$ & $-.30 * * *$ & .10 & .11 \\
\hline 7 Oración & & & & & & & $-.18 *$ & $.42 * *$ & $.30 * *$ & $.18 *$ & .15 & $-.56 * *$ & $.35^{* *}$ & -.09 & $.26^{* *}$ & .08 \\
\hline 8 Racismo Moderno & & & & & & & & .09 & .02 & $.34 * *$ & .16 & $.26^{* *}$ & $.31 * *$ & $-.45 * * *$ & $-.44 * *$ & .06 \\
\hline 9 Homofobia & & & & & & & & & .15 & $.32 * *$ & .11 & $-.30 * *$ & $.66^{* *}$ & $-.36 * * *$ & -.08 & .10 \\
\hline 10 Sexismo & & & & & & & & & & $.18 *$ & $.43 * *$ & -.10 & $.60 * *$ & -.12 & .12 & -.06 \\
\hline 11 Distancia & & & & & & & & & & & $.23 * *$ & -.10 & $.77 * *$ & $-.87 * * *$ & $-.60^{* * *}$ & $.27 * *$ \\
\hline 12 Dogmatismo & & & & & & & & & & & & -.07 & $.35 * *$ & $-.17 *$ & -.08 & .08 \\
\hline 13 Literalismo & & & & & & & & & & & & & $-.18 *$ & .05 & -.08 & $-.22 * *$ \\
\hline 14 Prejuicio Religioso & & & & & & & & & & & & & & $-.72 * * *$ & $-.35^{* * *}$ & .14 \\
\hline 15 Creencias & & & & & & & & & & & & & & & $.67 * *$ & $-.28 * *$ \\
\hline 16 Favorabilidad & & & & & & & & & & & & & & & & -.16 \\
\hline
\end{tabular}

$* p<.05 ; * * p<.01 ; * * * p<.001$.

El fundamentalismo correlaciona negativamente con creencias pero no con el racismo moderno tal como sucedía en la muestra universitaria. Se mantiene su correlación positiva con sexismo, homofobia, distancia social y prejuicio religioso, presentando una correlación negativa con la intolerancia religiosa.

La sumisión y agresión autoritaria correlaciona positivamente con conservadurismo, creencias, homofobia, sexismo, distancia social y prejuicio religioso. El conservadurismo correlaciona negativamente con creencias y favorabilidad y positivamente con homofobia, intolerancia, distancia social y prejuicio religioso. Por tanto, se encuentra una relación clara entre autoritarismo de derechas y prejuicio.

En el análisis de regresión lineal se comprueba que la mayoría de variables religiosas tienen muy poco o ningún valor predictivo. Se aprecia que las variables fundamentalismo y autoritarismo son los mejores predictores del prejuicio.

\section{Muestra musulmana}

Se observa que las siguientes variables están correlacionadas positivamente entre sí: orientación extrínseca e intrínseca, religión subjetiva, fundamentalismo, sumisión y agresión autoritaria, conservadurismo y frecuencia de oración (Tabla 6). Se encuentran las siguientes excepciones: orientación extrínseca y frecuencia de oración, religión subjetiva y autoritarismo de derechas y sumisión y agresión autoritaria y frecuencia de oración. La orientación de búsqueda no correlaciona con la dimensión intrínseca ni la extrínseca y lo hace negativamente con la religión subjetiva, fundamentalismo, racismo moderno y dogmatismo, mostrando una orientación religiosa distinta al resto. El literalismo religioso correlaciona con el autoritarismo de derechas y el fundamentalismo. 
Tabla 6. Correlaciones de la muestra musulmana extraída de centros de culto.

\begin{tabular}{|c|c|c|c|c|c|c|c|c|c|c|c|c|c|c|c|}
\hline & 2 & 3 & 4 & 5 & 6 & 7 & 8 & 9 & 10 & 11 & 12 & 13 & 14 & 15 & Extrínseca \\
\hline 1 Intrínseca & -.06 & $.18^{* *}$ & $.29 * * *$ & $-.22 * *$ & $.41^{* * *}$ & $.27^{* * *}$ & -.10 & $.17^{*}$ & $.17^{* *}$ & $-.36 * * *$ & $-.23^{* *}$ & -.10 & $.26 * * *$ & $.28 * * *$ & $.45^{* * *}$ \\
\hline 2 Búsqueda & & $-.15 * *$ & .05 & -.03 & $-.18 * *$ & .07 & $-.22 * *$ & .13 & .07 & .01 & $-.22^{* *}$ & .00 & .12 & $.18^{* * *}$ & .08 \\
\hline 3 Religión subjetiva & & & -.05 & -.10 & $.21^{* *}$ & $.32^{* * * *}$ & .00 & -.12 & -.09 & .12 & $.14 *$ & -.05 & $-.18^{* *}$ & $-.13 *$ & $.17^{* *}$ \\
\hline 4 Autoritarismo & & & & $-.33 * * *$ & $.45 * * *$ & .01 & $-.23 * *$ & -.03 & -.05 & -.03 & .03 & $-.14 *$ & -.02 & .13 & $.45^{* * *}$ \\
\hline 5 Conservadurismo & & & & & $-.39 * * *$ & $-.37 * * *$ & $.23 * *$ & -.07 & $.27 * * *$ & -.08 & $.15^{*}$ & $.30 * * *$ & .10 & .08 & $-.29 * * *$ \\
\hline 6 Fundamentalismo & & & & & & $.25 * * *$ & $-.18 * *$ & .09 & -.05 & -.07 & .02 & $-.32 * * *$ & -.05 & .09 & $.51^{* * *}$ \\
\hline 7 Oración & & & & & & & -.06 & .12 & -.04 & -.01 & -.11 & -.12 & .02 & -.02 & .12 \\
\hline 8 Racismo Moderno & & & & & & & & .13 & $.23 * *$ & .12 & $.21 * *$ & .06 & $-.26 * * *$ & $-.35^{* * *}$ & $-.28 * * *$ \\
\hline 9 Homofobia & & & & & & & & & $.72 * *$ & $-.41 * *$ & $-.28 * *$ & $-.25^{* *}$ & $.45^{* * *}$ & $.24 * * *$ & $-.19 * *$ \\
\hline 10 Sexismo & & & & & & & & & & $-.41 * *$ & $-.26^{* *}$ & -.13 & $.47 * * *$ & $.26^{* * *}$ & -.22 \\
\hline 11 Distancia & & & & & & & & & & & $.25^{* *}$ & .05 & $-.79 * * *$ & $-.61^{* * *}$ & -.03 \\
\hline 12 Dogmatismo & & & & & & & & & & & & -.01 & $-.37 * * *$ & $-.37 * * *$ & .02 \\
\hline 13 Literalismo & & & & & & & & & & & & & -.07 & -.02 & -.07 \\
\hline 14 Creencias & & & & & & & & & & & & & & $.62 * *$ & -.07 \\
\hline 15 Favorabilidad & & & & & & & & & & & & & & & .10 \\
\hline
\end{tabular}

$*_{p}<.05 ; * * p<.01 ; * * * p<.001$.

Además, la orientación intrínseca correlaciona positivamente con homofobia y sexismo y negativamente con distancia social e intolerancia religiosa lo que ayuda a explicar por qué la escala del prejuicio religioso no tiene validez en la muestra musulmana.

El fundamentalismo correlaciona negativamente con racismo moderno y positivamente con el literalismo religioso y los derechos de gays y lesbianas. Además, la sumisión y agresión autoritaria correlaciona negativa e inesperadamente con emociones negativas, racismo moderno e intolerancia y positivamente con los derechos de gays y lesbianas. El conservadurismo sólo correlaciona negativamente con racismo moderno y sexismo y positivamente con los derechos de gays y lesbianas. En conclusión, el autoritarismo de derechas no parece afectar al desarrollo del prejuicio hacia los cristianos.

En el análisis de regresión lineal se obtiene que el dogmatismo se presenta como la única variable con cierto valor predictivo para la variable creencias $\left(R^{2}=.14\right)$ seguido de la dimensión intrínseca. Para el racismo moderno, la orientación extrínseca es el "mejor", aunque pobre, predictor $\left(R^{2}=.08\right)$. Por otra parte, el autoritarismo de derechas no muestra ser un buen predictor para ninguna de las variables consideradas.

Diferencias por religión y entre los estudios $1^{\circ}$ y $2^{\circ}$

Se realiza un conjunto de análisis de varianza ANOVA con dos factores: religión y grupo para analizar las diferencias entre los cuatro grupos estudiados (Tabla 4). Al utilizar como variable dependiente el racismo moderno, se encuentran diferencias significativas por religión y grupo; existen diferencias significativas entre cristianos universitarios y pertenecientes a grupos religiosos $(p<.001)$ pero no entre los musulmanes universitarios y los extraídos fuera de la universidad. Si la variable dependiente son las emociones positivas, se obtienen diferencias significativas por religión, grupo y en la intersección de ambas.
Utilizando las emociones negativas, se constatan diferencias significativas por religión y con creencias se observan diferencias por grupo y religión.

Al utilizar variables de religiosidad como la frecuencia de oración o la religión subjetiva como variables dependientes, se observa que las diferencias se encuentran, en ambos casos, en la religión, en el grupo y en la interacción del grupo con la religión. Así, los cristianos rezan menos $(p<.001)$ y puntúan menos en religión subjetiva $(p<.001)$. Realizando sendos ajustes por comparaciones múltiples de Bonferroni, existen diferencias significativas entre universitarios y no universitarios en cristianos $(p<.001, p<.001)$ para ambas variables y para los musulmanes $(p<.001)$ sólo en la frecuencia de oración. Al realizar el ANOVA con la dimensión intrínseca, los cristianos puntúan menos $(p<.001)$; además, los cristianos universitarios son menos intrínsecos que sus pares no universitarios $(p<.001)$ pero no sucede lo mismo con la muestra musulmana.

En literalismo se encuentran diferencias significativas por religión y grupo. Los cristianos interpretan menos literalmente los textos sagrados que los musulmanes $(p<.001)$. En esta variable existen diferencias significativas entre universitarios y no universitarios sólo en los cristianos $(p<.001)$. Con el fundamentalismo como variable dependiente, los factores incluidos en el modelo explican un $57 \%$ de la varianza de la variable dependiente, siendo además significativas las diferencias de religión y grupo, así como, la interacción entre ambas. Los cristianos son menos fundamentalistas $(p<.001)$ y los universitarios menos que sus pares no universitarios, tanto en musulmanes $(p<.001)$ como en cristianos $(p<.001)$.

En el autoritarismo de derechas, el análisis muestra que las diferencias se encuentran en la religión y en el grupo. Los cristianos son menos autoritarios y conservadores que los musulmanes y los universitarios menos que los no universitarios, tanto en cristianos como musulmanes. 
Finalmente, respecto a la homofobia, el ANOVA muestra que los factores (religión, grupo, grupo*religión) explican el $37 \%$ de la varianza de la variable dependiente y que las diferencias se encuentran en la religión y en el grupo. Los cristianos son menos homófobos $(p<.001)$ y los universitarios más, si bien con una significación débil, tanto en cristianos $(p=.03)$ como en musulmanes ( $p=.03$ ). Si se utiliza el factor "derechos de gays y lesbianas", el análisis revela diferencias por grupo, religión y la interacción de ambos. En todo caso, los musulmanes son más homófobos que los cristianos $(p<.001)$ y los universitarios, de forma opuesta al otro factor, son menos homófobos: cristianos $(p<.001)$ y musulmanes $(p<.001)$.

Respecto al sexismo benevolente como variable dependiente se obtiene que la religión, el grupo y la interacción entre ambos sólo explican un 3\% de la variable dependiente. Sólo es significativa la interacción entre religión y grupo. Existen diferencias significativas, pero en sentido contrario, entre universitarios y no universitarios para los cristianos $(p<.01)$ y musulmanes $(p<.001)$. Sin embargo, los musulmanes no son más sexistas que los cristianos.

\section{Discusión}

En primer lugar, nuestro instrumento, Escala del Prejuicio Religioso, se muestra como un instrumento útil para conocer la compleja relación religión prejuicio en la población cristiana. No sólo presenta buenas propiedades psicométricas sino que correlaciona positivamente con la orientación intrínseca y la religión subjetiva, tanto en la población universitaria como no universitaria, así como, con las variables mediadoras: autoritarismo de derechas, fundamentalismo, dogmatismo y literalismo. Estas correlaciones están en la línea de diversos estudios que relacionan la religiosidad o al menos sus formas más conservadoras y ortodoxas a la homofobia, el sexismo y la intolerancia religiosa (Ellis, Kitzinger y Wilkinson, 2002; Hunsberger y Jackson, 2005; Rosik, 2007). Sin embargo, no podemos avalar la Escala del Prejuicio Religioso en el caso musulmán, el problema se presenta fundamentalmente en que el factor homofobia es completamente distinto al resto. Así, Boellstroff (2005) afirma que ser gay en Indonesia es "inconmensurable" y Abdelwahad Bouhdiba apostilla: "En el Islam la homosexualidad masculina representa todas las perversiones y constituye en este sentido la depravación de las depravaciones" (Bouhdiba, 1998, p. 31).

Por otra parte, las variables de autoritarismo de derechas y el fundamentalismo son predictores del prejuicio en la muestra cristiana (Laythe et al., 2002). En los musulmanes, emergen como predictores el dogmatismo y las orientaciones extrínseca e intrínseca; así, el dogmatismo parece captar más la rigidez del pensamiento y la intolerancia y ser menos dependiente del contexto sociopolítico.

Además, se aprecia que en líneas generales, tanto los musulmanes como los no universitarios de ambas religiones son más religiosos, fundamentalistas, autoritarios e interpretan más literalmente los textos sagrados. Además, son más homófobos pero no más sexistas. Conviene señalar que el estudio de las variables religiosas y de otras variables intervinientes como el fundamentalismo, el literalismo y el autoritarismo de derechas debe contextualizarse en una sociedad concreta. España y Marruecos, éste último como país referente para la mayoría de los encuestados musulmanes, se encuentran, a pesar de su proximidad geográfica, inmersos en procesos sociales, políticos, económicos y religiosos completamente diferentes.

Los musulmanes interpretan más literalmente los textos sagrados que los cristianos. Esto puede explicarse en la evolución de la Iglesia Católica a partir del Concilio Vaticano II; así, la Dei Verbum, se manifiesta claramente en contra de una interpretación literal de la Biblia. Sin embargo, los musulmanes puntúan muy alto en literalismo, tanto los universitarios como los no universitarios. Nuestros resultados avalan los estudios de Davis y Robinson (2006) que aseguran que al preguntar si el Corán o la Biblia son revelados divinamente, infalibles $\mathrm{y}$ deben ser tomados literalmente, se consigue discriminar en otros credos religiosos pero no en los musulmanes.

Finalmente, se observa que los cristianos puntúan más alto en la orientación de búsqueda. Ésta se manifiesta como una dimensión completamente distinta al resto de las orientaciones y variables religiosas. Se puede interpretar como una predisposición a buscar respuestas, con una mentalidad tolerante y abierta, a las grandes preguntas existenciales de la vida o, tal vez, cierta indiferencia e incluso rechazo a la religión (Watson et al., 1999).

Este trabajo de investigación aporta a la Psicología de la Religión un análisis discriminante circunscrito en dos de las grandes religiones: cristianismo e islam, ésta última bastante ausente en la literatura. Además, no nos hemos limitado a la muestra "típica" de estudiantes universitarios, sino también hemos acudido a centros de culto para tratar de conseguir unos resultados menos "de laboratorio", más fácilmente generalizables. Sin embargo, se aprecian ciertas limitaciones; en particular, debe ponderarse el componente ideológico subyacente, lo cual exigirá en estudios posteriores determinar las diferencias en función del grupo religioso. Grupos con características ideológicas diversas que, a veces, diferencian enormemente su ser religioso, aunque posean teóricamente la misma fe.

\section{Referencias}

Allport, G. W. y Ross, J. M. (1967). Personal religious orientation and prejudice. Journal of Personality \& Social Psychology, 5, 432-443.

Altemeyer, B. (1996). The authoritarian specter. Cambridge, MA: Harvard University Press.

Altemeyer, B. y Hunsberger, B. (2004). A revised religious fundamentalism scale: The short and the sweet of it. The 
International Journal for the Psychology of Religion, 14, 47-54.

Aosved, A. C. y Long, P. J. (2006). Co-occurrence of Rape Myth Acceptance, Sexism, Racism, Homophobia, Ageism, Classism, and Religious Intolerance. Sex Roles 55, 481-492.

Batson, C. D. y Schoenrade, P. (1991). Measuring religion as quest: 1. Validity concerns. Journal for the Scientific Study of Religion, 30, 416-429.

Batson, C. D., Schoenrade, P. y Ventis, W. L. (1993). Religion and the individual: A social-psychological perspective. New York: Oxford University Press.

Batson, C. D. y Ventis, W. L. (1982). The religious experience: A social-psychological perspective. New York: Oxford University Press.

Boellstroff, T. (2005). Between Religion and Desire: Being Muslim and Gay in Indonesia. American Anthropologist, 107, 575-585.

Bouhdiba, A. (1998). Sexuality in Islam. London: Saqi Books.

Brigham, J. C. (1993). College students' racial attitudes. Journal of Applied Social Psychology, 23, 1933-1977.

CIRES (1993). La realidad social en España 1991-92. Barcelona: Ediciones B.

Davis, N. J. y Robinson, R. (2006). The Egalitarian Face of Orthodox Islam: Support for Islamic Law and Equality in Seven Muslim-Majority Nations. American Sociological Review, 71, 167-190.

Ellis, S. J., Kitzinger, C. y Wilkinson, S. (2002). Attitudes towards lesbians and gay men and support for lesbian and gay human rights among psychology students. Journal of homosexuality, 44, 121-138.

García, M. C., Navas, M. S., Cuadrado, I. y Molero, F. (2003). Inmigración y prejuicio: Actitudes de una muestra de adolescentes almerienses. Acción Psicológica, 2, 137-147.

Gay, D. y Lynxwiler, J. (1999). The impact of religiosity on race variations in abortion attitudes. Sociological Spectrum, 19, 359-377.

Glick, P. y Fiske, S. T. (1996). The Ambivalent Sexism Inventory: Differentiating hostile and benevolent sexism. Journal of Personality and Social Psychology, 70, 491-512.

Herek, G. M. (1998). The Attitudes Toward Lesbians and Gay Men (ATLG) scale. En C. M. Davis, W. H. Yarber, R. Bauserman, G. Schreer y S. L. Davis (Eds.), Sexualityrelated measures: A compendium (pp. 392-394). Thousand Oaks, CA: Sage Publications.

Hood, R. W. Jr., Spilka, B., Hunsberger, B. y Gorsuch, R. L. (2009). The psychology of religion: An empirical approach ( $4^{\mathrm{a}}$ ed.). New York: The Guilford Press.

Hunsberger, B. y Jackson, L. M. (2005). Religion, Meaning, and Prejudice. Journal of Social Issues, 61, 807-826.

Laythe, B., Finkel, D. G., Bringle, R. B. y Kirkpatrick, L. A. (2002). Religious fundamentalism as a predictor of prejudice: A two-component model. Journal for the Scientific Study of Religion, 41, 623-635.
Levin, J., Taylor, R. y Chatters, L. (1995). A multidimensional measure of religious involvement for African Americans. The Sociological Quarterly, 36, 157-173.

Maltby, J., Lewis, C. A. y Day, L. (1999). Religious orientation and psychological well-being: The role of the frequency of personal prayer. British Journal of Health Psychology, 4, 363-378.

Manganelli Rattazzi, A. M., Bobbio, A. y Canova, L. (2007). A short version of the Right-Wing Authoritarianism (RWA) Scale. Personality and Individual Differences, 43, 12231234.

Mattis, J. S., Fontenot, D. L. y Hatcher-Kay, C. A. (2003). Religiosity, racism, and dispositional optimism among African Americans. Personality and Individual Differences, 34, 1025-1038.

Meadow, M. J. y Kahoe, R. D. (1985). Psychology of Religion: Religion in individual lives. New York: Harper \& Row.

Ramírez de la Fe, Ma C. (2006). Una adaptación española de la escala de orientación religiosa de Batson y Ventis. Revista de Psicología General y Aplicada, 59, 309-318.

Rizzo, H., Abdel-Latif, A. H. y Mejer, K. (2007). The Relationship Between Gender Equality and Democracy: A Comparison of Arab Versus Non-Arab Muslim Societies. Sociology, 41, 1151-1170.

Rosik, C.H.(2007). Ideological concern in the operationalization of homophobia, part I: An analysis of Herek's ATLG-R scale. Journal of Psychology and Theology, 35, 132-144.

Rowatt, C. W., LaBouff, J., Johnson, M., Froese, P. y Tsang, J. (2009). Associations among religiousness, social attitudes, and prejudice in a national random sample of American adults. Psychology of Religion and Spirituality, 1, 14-24.

Stephan, W. G. y Stephan, C. W. (1985). Intergroup anxiety. Journal of Social Issues, 41, 157- 175.

Taylor, S.J. y Bogdan, R. (1986). Introducción a los métodos cualitativos de investigación. La búsqueda de significados. Barcelona: Editorial Paidós.

Taylor, R., Mattis, J. y Chatters, L. (1999). Subjective religiosity among African Americans: a synthesis of findings from five national samples. Journal of Black Psychology, 25, 524 543.

Tessler, M. (2003). Arab and Muslim political attitudes: Stereotypes and evidence from survey research. International Studies Perspectives, 4, 175-180.

Tropp, L. R. y Pettigrew, T. F. (2005). Differential Relationships Between Intergroup Contact and Affective and Cognitive Dimensions of Prejudice. Personality and Social Psychology Bulletin, 31, 1145-1158.

Tsang, J. y Mak, H. K. (2008). Separating the "Sinner" from the "Sin": Religious Orientation and Prejudice toward Sexual Orientation and Promiscuous Sex. Journal for the Scientific Study of Religion, 47, 379-392.

Tsang, J. y Rowatt, W. C. (2007). The Relationship Between Religious Orientation, Right-Wing Authoritarianism, and 
Implicit Sexual Prejudice. The International Journal for the Psychology of Religion, 17, 99-120.

Watson, P. J., Morris, R. J., Hood, R. W. Jr., Miller, L. y Waddell, M. G. (1999). Religion and the experiential system: Relationships of constructive thinking with religious orientation. The International Journal for the Psychology of Religion, 9, 195-207.

Whitley, B. E. (2009). Religiosity and Attitudes Toward Lesbians and Gay Men: A Meta-Analysis. International Journal for the Psychology of Religion, 19, 21 - 38.
Wulff, D. (1997). Psychology of Religion: Classic and contemporary ( $2^{\mathrm{a}}$ ed.). Oxford, England: John Wiley and Sons.

Fecha de recepción: 26 de abril de 2010

Fecha de recepción de la primera versión modificada: 28 de junio de 2010

Fecha de recepción de la segunda versión modificada: 21 de septiembre de 2010

Fecha de aceptación: 29 de septiembre de 2010 